\title{
Cellular Automata on Group Sets and the Uniform Curtis-Hedlund-Lyndon Theorem
}

\author{
Simon Wacker \\ Karlsruhe Institute of Technology \\ simon.wacker@kit.edu \\ http://www.kit.edu
}

\begin{abstract}
We introduce cellular automata whose cell spaces are left homogeneous spaces and prove a uniform as well as a topological variant of the Curtis-Hedlund-Lyndon theorem. Examples of left homogeneous spaces are spheres, Euclidean spaces, as well as hyperbolic spaces acted on by isometries; vertex-transitive graphs, in particular, Cayley graphs, acted on by automorphisms; groups acting on themselves by multiplication; and integer lattices acted on by translations.
\end{abstract}

Keywords: cellular automata, group actions, Curtis-Hedlund-Lyndon theorem

In the first chapter of the monograph 'Cellular Automata and Groups' [1], Tullio Ceccherini-Silberstein and Michel Coornaert develop the theory of cellular automata whose cell spaces are groups. Examples of groups are abound: The integer lattices and Euclidean spaces with addition (translation), the onedimensional unit sphere embedded in the complex plane with complex multiplication (rotation), and the vertices of a Cayley graph with the group structure it encodes (graph automorphisms).

Yet, there are many structured sets that do not admit a structure-preserving group structure. For example: Each Euclidean $n$-sphere, except for the zero-, one-, and three-dimensional, does not admit a topological group structure; the Petersen graph is not a Cayley graph and does thus not admit an edge-preserving group structure on its vertices. However, these structured sets can be acted on by subgroups of their automorphism group by function application. For example Euclidean $n$-spheres can be acted on by rotations about the origin and graphs can be acted on by edge-preserving permutations of their vertices.

Moreover, there are structured groups that have more symmetries than can be expressed with the group structure. The integer lattices and the Euclidean spaces with addition, for example, are groups, but addition expresses only their translational symmetries and not their rotational and reflectional ones. Though, they can be acted on by arbitrary subgroups of their symmetry groups, like the ones generated by translations and rotations.

The general notion that encompasses these structure-preserving actions is that of a group set, that is, a set that is acted on by a group. A group set $M$ acted on by $G$ such that for each tuple $\left(m, m^{\prime}\right) \in M \times M$ there is a symmetry 
$g \in G$ that transports $m$ to $m^{\prime}$ is called left homogeneous space and the action of $G$ on $M$ is said to be transitive. In particular, groups are left homogeneous spaces - they act on themselves on the left by multiplication.

In this paper, we develop the theory of cellular automata whose cell spaces are left homogeneous spaces which culminates in the proof of a uniform and topological variant of a famous theorem by Morton Landers Curtis, Gustav Arnold Hedlund, and Roger Conant Lyndon from 1969, see the paper 'Endomorphisms and automorphisms of the shift dynamical system' 22. The development of this theory is greatly inspired by [1].

These cellular automata are defined so that their global transition functions are equivariant under the induced group action on global configurations. Depending on the choice of the cell space, these actions may be plain translations but also rotations and reflections. Exemplary for the first case are integer lattices that are acted on by translations; and for the second case Euclidean $n$-spheres that are acted on by rotations, but also the two-dimensional integer lattice that is acted on by the group generated by translations and the rotation by $90^{\circ}$.

Sébastien Moriceau defines and studies a more restricted notion of cellular automata over group sets in his paper 'Cellular Automata on a $G$-Set' [3]. He requires sets of states and neighbourhoods to be finite. His automata are the global transition functions of, what we call, semi-cellular automata with finite set of states and finite essential neighbourhood.

His automata obtain the next state of a cell by shifting the global configuration such that the cell is moved to the origin, restricting that configuration to the neighbourhood of the origin, and applying the local transition function to that local configuration. Our automata obtain the next state of a cell by determining the neighbours of the cell, observing the states of that neighbours, and applying the local transition function to that observed local configuration. The results are the same but the viewpoints are different, which manifests itself in proofs and constructions.

To determine the neighbourhood of a cell we let the relative neighbourhood semi-act on the right on the cell. That right semi-action is to the left group action what right multiplication is to the corresponding left group multiplication. Many properties of cellular automata are a consequence of the interplay between properties of that semi-action, shifts of global configurations, and rotations of local configurations. That semi-action also plays an important role in our definition of right amenable left group sets, see [5], for which the Garden of Eden theorem holds, see [6], which states that each cellular automaton is surjective if and only if it is pre-injective. For example finitely right generated left homogeneous spaces of sub-exponential growth are right amenable, in particular, quotients of finitely generated groups of sub-exponential growth by finite subgroups acted on by left multiplication.

In Sect. 1 we introduce left group actions and our prime example, which illustrates phenomena that cannot be encountered in groups acting on themselves on the left by multiplication. In Sect.2 we introduce coordinate systems, cell spaces, and right quotient set semi-actions that are induced by left group actions and 
coordinate systems. In Sect. 3 we introduce semi-cellular and cellular automata. In Sect. [ we show that a global transition function does not depend on the choice of coordinate system, is equivariant under the induced left group action on global configurations, is determined by its behaviour in the origin, and that the composition of two global transition functions is a global transition function. In Sect. 5 we prove a uniform and a topological variant of the Curtis-HedlundLyndon theorem, which characterise global transition functions of semi-cellular automata by uniform and topological properties respectively. And in Sect. 6 we characterise invertibility of semi-cellular automata.

\section{Left Group Actions}

Definition 1. Let $M$ be a set, let $G$ be a group, let $\triangleright$ be a map from $G \times M$ to $M$, and let $e_{G}$ be the neutral element of $G$. The map $\triangleright$ is called left group action of $G$ on $M$, the group $G$ is said to act on $M$ on the left by $\triangleright$, and the triple $(M, G, \triangleright)$ is called left group set if and only if

$$
\begin{gathered}
\forall m \in M: e_{G} \triangleright m=m, \\
\forall m \in M \forall g \in G \forall g^{\prime} \in G: g g^{\prime} \triangleright m=g \triangleright\left(g^{\prime} \triangleright m\right) .
\end{gathered}
$$

Example 1. Let $G$ be a group. It acts on itself on the left by $\triangleright$ by multiplication.

Example 2. Let $M$ be the Euclidean unit 2-sphere, that is, the surface of the ball of radius 1 in 3-dimensional Euclidean space, and let $G$ be the rotation group. The group $G$ acts on $M$ on the left by $\triangleright$ by function application, that is, by rotation about the origin.

Definition 2. Let $\triangleright$ be a left group action of $G$ on $M$ and let $H$ be a subgroup of $G$. The left group action $\triangleright \uparrow_{H \times M}$ of $H$ on $M$ is denoted by $\triangleright_{H}$.

Definition 3. Let $\triangleright$ be a left group action of $G$ on $M$. It is called

1. transitive if and only if the set $M$ is non-empty and

$$
\forall m \in M \forall m^{\prime} \in M \exists g \in G: g \triangleright m=m^{\prime} ;
$$

2. free if and only if

$$
\forall g \in G \forall g^{\prime} \in G:\left(\exists m \in M: g \triangleright m=g^{\prime} \triangleright m\right) \Longrightarrow g=g^{\prime} .
$$

Example 3. In the situation of Example 1, the left group action is transitive and free.

Example 4. In the situation of Example 2, the left group action is transitive but not free.

Definition 4. Let $(M, G, \triangleright)$ be a left group set. It is called left homogeneous space if and only if the left group action $\triangleright$ is transitive. 
Definition 5. Let $\triangleright$ be a left group action of $G$ on $M$, and let $m$ and $m^{\prime}$ be two elements of $M$.

1. The set $G \triangleright m=\{g \triangleright m \mid g \in G\}$ is called orbit of $m$ under $\triangleright$.

2. The set $G_{m}=\{g \in G \mid g \triangleright m=m\}$ is called stabiliser of $m$ under $\triangleright$.

3. The set $G_{m, m^{\prime}}=\left\{g \in G \mid g \triangleright m=m^{\prime}\right\}$ is called transporter of $m$ to $m^{\prime}$ under $\triangleright$.

Example 5. In the situation of Example 3, each orbit is $G$ and each stabiliser is $\left\{e_{G}\right\}$.

Example 6. In the situation of Example 4, for each point $m \in M$, its orbit is $M$ and its stabiliser is the group of rotations about the line through the origin and itself.

Lemma 1. Let $\triangleright$ be a left group action of $G$ on $M$, let $m$ and $m^{\prime}$ be two elements of $M$ that have the same orbit under $\triangleright$, and let $g$ be an element of $G_{m, m^{\prime}}$. Then, $G_{m^{\prime}}=g G_{m} g^{-1}$ and $g G_{m}=G_{m, m^{\prime}}=G_{m^{\prime}} g$.

Proof. First, let $g^{\prime} \in G_{m}$. Then,

$$
g g^{\prime} g^{-1} \triangleright m^{\prime}=g g^{\prime} \triangleright m=g \triangleright m=m^{\prime} .
$$

Hence, $g g^{\prime} g^{-1} \in G_{m^{\prime}}$. In conclusion, $G_{m^{\prime}} \supseteq g G_{m} g^{-1}$. Secondly, let $g^{\prime} \in G_{m^{\prime}}$. Then, as above, $g^{\prime \prime}=g^{-1} g^{\prime} g \in G_{m}$. Hence, $g^{\prime}=g g^{\prime \prime} g^{-1} \in g G_{m} g^{-1}$. In conclusion, $G_{m^{\prime}} \subseteq g G_{m} g^{-1}$. To sum up, $G_{m^{\prime}}=g G_{m} g^{-1}$. Moreover, $g G_{m}=G_{m^{\prime}} g$.

Thirdly, let $g^{\prime} \in G_{m}$. Then, $g g^{\prime} \in G_{m, m^{\prime}}$. In conclusion, $g G_{m} \subseteq G_{m, m^{\prime}}$. Lastly, let $g^{\prime} \in G_{m, m^{\prime}}$. Then, $g^{\prime \prime}=g^{-1} g^{\prime} \in G_{m}$. Hence, $g^{\prime}=g g^{\prime \prime} \in g G_{m}$. In conclusion, $g G_{m} \supseteq G_{m, m^{\prime}}$. To sum up, $g G_{m}=G_{m, m^{\prime}}$.

Definition 6. Let $M$ and $M^{\prime}$ be two sets, let $f$ be a map from $M$ to $M^{\prime}$, and let $\triangleright$ be a left group action of $G$ on $M$. The map $f$ is called $\triangleright$-invariant if and only if

$$
\forall g \in G \forall m \in M: f(g \triangleright m)=f(m) .
$$

Definition 7. Let $M$ and $M^{\prime}$ be two sets, let $f$ be a map from $M$ to $M^{\prime}$, and let $\triangleright$ and $\triangleright^{\prime}$ be two left group actions of $G$ on $M$ and $M^{\prime}$ respectively. The map $f$ is called $\left(\triangleright, \triangleright^{\prime}\right)$-equivariant if and only if

$$
\forall g \in G \forall m \in M: f(g \triangleright m)=g \triangleright^{\prime} f(m) ;
$$

and $\triangleright$-equivariant if and only if it is $\left(\triangleright, \triangleright^{\prime}\right)$-equivariant, $M=M^{\prime}$, and $\triangleright=\triangleright^{\prime}$.

Lemma 2. Let $f$ be a $\left(\triangleright, \triangleright^{\prime}\right)$-equivariant and bijective map from $M$ to $M^{\prime}$. The inverse of $f$ is $\left(\triangleright^{\prime}, \triangleright\right)$-equivariant

Proof. For each $g \in G$ and each $m \in M$,

$$
f^{-1}(g \triangleright m)=f^{-1}\left(g \triangleright f\left(f^{-1}(m)\right)\right)=f^{-1}\left(f\left(g \triangleright f^{-1}(m)\right)\right)=g \triangleright f^{-1}(m) .
$$


Lemma 3. Let $G$ be a group and let $H$ be a subgroup of $G$. The group $G$ acts transitively on the quotient set $G / H$ on the left by

$$
\begin{aligned}
\because G \times G / H & \rightarrow G / H, \\
\left(g, g^{\prime} H\right) & \mapsto g g^{\prime} H .
\end{aligned}
$$

Proof. The map · is well-defined, because, for each $g \in G$, each $g_{1}^{\prime} \in G$, and each $g_{2}^{\prime} \in G$,

$$
g_{1}^{\prime} H=g_{2}^{\prime} H \Longleftrightarrow g g_{1}^{\prime} H=g g_{2}^{\prime} H .
$$

It is a left group action, because, for each $g^{\prime} H \in G / H$,

$$
e_{G} \cdot g^{\prime} H=g^{\prime} H,
$$

and, for each $g_{1} \in G$, each $g_{2} \in G$, and each $g^{\prime} H \in G / H$,

$$
\begin{aligned}
g_{1} g_{2} \cdot g^{\prime} H & =g_{1} g_{2} g^{\prime} H \\
& =g_{1} \cdot g_{2} g^{\prime} H \\
& =g_{1} \cdot\left(g_{2} \cdot g^{\prime} H\right) .
\end{aligned}
$$

It is transitive, because, for each $g_{1}^{\prime} H \in G / H$ and each $g_{2}^{\prime} H \in G / H$,

$$
g_{2}^{\prime} g_{1}^{\prime-1} \cdot g_{1}^{\prime} H=g_{2}^{\prime} H .
$$

Lemma 4. Let $\triangleright$ be a transitive left group action of $G$ on $M$, let $m_{0}$ be an element of $M$, and let $G_{0}$ be the stabiliser of $m_{0}$ under $\triangleright$. The map

$$
\begin{aligned}
\iota: M & \rightarrow G / G_{0}, \\
m & \mapsto G_{m_{0}, m},
\end{aligned}
$$

is $(\triangleright, \cdot)$-equivariant and bijective.

Proof. For each $g \in G$ and each $m \in M$,

$$
\iota(g \triangleright m)=G_{m_{0}, g \triangleright m}=g \cdot G_{m_{0}, m}=g \cdot \iota(m) .
$$

Hence, $\iota$ is $(\triangleright, \cdot)$-equivariant. Moreover, for each $\left(m, m^{\prime}\right) \in M \times M$ with $m \neq m^{\prime}$, we have $G_{m_{0}, m} \neq G_{m_{0}, m^{\prime}}$. Thus, $\iota$ is injective. Furthermore, for each $g G_{0} \in G /$ $G_{0}$, we have $G_{m_{0}, g \triangleright m_{0}}=g G_{0}$. Therefore, $\iota$ is surjective.

\section{Right Quotient Set Semi-Actions}

Definition 8. Let $\mathcal{M}=(M, G, \triangleright)$ be a left homogeneous space, let $m_{0}$ be an element of $M$, let $g_{m_{0}, m_{0}}$ be the neutral element of $G$, and, for each element $m \in M \backslash\left\{m_{0}\right\}$, let $g_{m_{0}, m}$ be an element of $G$ such that $g_{m_{0}, m} \triangleright m_{0}=m$. The tuple $\mathcal{K}=\left(m_{0},\left\{g_{m_{0}, m}\right\}_{m \in M}\right)$ is called coordinate system for $\mathcal{M}$; the element $m_{0}$ is called origin; for each element $m \in M$, the element $g_{m_{0}, m}$ is called coordinate of $m$; for each subgroup $H$ of $G$, the stabiliser of the origin $m_{0}$ under $\triangleright_{H}$, which is $G_{m_{0}} \cap H$, is denoted by $H_{0}$. 
Definition 9. Let $\mathcal{M}=(M, G, \triangleright)$ be a left homogeneous space and let $\mathcal{K}=\left(m_{0}\right.$, $\left.\left\{g_{m_{0}, m}\right\}_{m \in M}\right)$ be a coordinate system for $\mathcal{M}$. The tuple $\mathcal{R}=(\mathcal{M}, \mathcal{K})$ is called cell space, each element $m \in M$ is called cell, and each element $g \in G$ is called symmetry.

Example \%. In the situation of Example 5 let $m_{0}$ be the neutral element $e_{G}$ of $G$ and, for each element $m \in G$, let $g_{m_{0}, m}$ be the only element in $G$ such that $g_{m_{0}, m} m_{0}=m$, namely $m$. The tuple $\mathcal{K}=\left(m_{0},\left\{g_{m_{0}, m}\right\}_{m \in M}\right)$ is a coordinate system of $\mathcal{M}=(G, G, \cdot)$ and the tuple $\mathcal{R}=(\mathcal{M}, \mathcal{K})$ is a cell space.

Example 8. In the situation of Example [6 let $m_{0}$ be the north pole $(0,0,1)^{\top}$ of $M$ and, for each point $m \in M$, let $g_{m_{0}, m}$ be a rotation about an axis in the $(x, y)$-plane that rotates $m_{0}$ to $m$. Note that $g_{m_{0}, m_{0}}$ is the trivial rotation. The tuple $\mathcal{K}=\left(m_{0},\left\{g_{m_{0}, m}\right\}_{m \in M}\right)$ is a coordinate system of $\mathcal{M}=(M, G, \triangleright)$ and the tuple $\mathcal{R}=(\mathcal{M}, \mathcal{K})$ is a cell space.

In the remainder of this section, let $\mathcal{R}=\left((M, G, \triangleright),\left(m_{0},\left\{g_{m_{0}, m}\right\}_{m \in M}\right)\right)$ be a cell space.

Lemma 5. The map

$$
\begin{aligned}
\varangle: M \times G / G_{0} & \rightarrow M, \\
\left(m, g G_{0}\right) & \mapsto g_{m_{0}, m} g g_{m_{0}, m}^{-1} \triangleright m\left(=g_{m_{0}, m} g \triangleright m_{0}\right),
\end{aligned}
$$

is a right quotient set semi-action of $G / G_{0}$ on $M$ with defect $G_{0}$, which means that, for each subgroup $H$ of $G$ such that $\left\{g_{m_{0}, m} \mid m \in M\right\} \subseteq H$,

$$
\forall m \in M: m \preccurlyeq G_{0}=m,
$$

$\forall m \in M \forall h \in H \exists h_{0} \in H_{0}: \forall \mathfrak{g} \in G / G_{0}: m \leqslant h \cdot \mathfrak{g}=\left(m \leqslant h G_{0}\right) \preccurlyeq h_{0} \cdot \mathfrak{g}$.

Proof. For each $m \in M$,

$$
m \varangle G_{0}=m \varangle e_{G} G_{0}=g_{m_{0}, m} e_{G} \triangleright m_{0}=g_{m_{0}, m} \triangleright m_{0}=m .
$$

Let $H$ be a subgroup of $G$ such that $\left\{g_{m_{0}, m} \mid m \in M\right\} \subseteq H$. Furthermore, let $m \in M$ and let $h \in H$. Put $h_{0}=g_{m_{0}, g_{m_{0}, m} h \triangleright m_{0}}^{-1} g_{m_{0}, m} h$. Because $g_{m_{0}, g_{m_{0}, m} h \triangleright m_{0}}^{-1} \in$ $H, g_{m_{0}, m} \in H$, and

$$
\begin{aligned}
h_{0} \triangleright m_{0} & =g_{m_{0}, g_{m_{0}, m} h \triangleright m_{0}}^{-1} \triangleright\left(g_{m_{0}, m} h \triangleright m_{0}\right) \\
& =m_{0},
\end{aligned}
$$

we have $h_{0} \in H_{0}$. Moreover, for each $g G_{0} \in G / G_{0}$,

$$
\begin{aligned}
m \star h \cdot g G_{0} & =m \varangle h g G_{0} \\
& =g_{m_{0}, m} h g \triangleright m_{0} \\
& =g_{m_{0}, g_{m_{0}, m} h \triangleright m_{0}} h_{0} g \triangleright m_{0} \\
& =\left(g_{m_{0}, m} h \triangleright m_{0}\right) \preccurlyeq h_{0} g G_{0} \\
& =\left(m \preccurlyeq h G_{0}\right) \preccurlyeq h_{0} \cdot g G_{0} .
\end{aligned}
$$


Example 9. In the situation of Example [7, the stabiliser $G_{0}$ of the neutral element $m_{0}$ under · is the trivial subgroup $\left\{e_{G}\right\}$ of $G$ and, for each element $g \in G$, we have $g_{m_{0}, m} g g_{m_{0}, m}^{-1} m=m g m^{-1} m=m g$. Under the natural identification of $G / G_{0}$ with $G$, the induced semi-action $\star$ is the right group action of $G$ on itself by multiplication.

Example 10. In the situation of Example8, the stabiliser $G_{0}$ of the north pole $m_{0}$ under $\triangleright$ is the group of rotations about the $z$-axis. An element $g G_{0} \in G / G_{0}$ semiacts on a point $m$ on the right by the induced semi-action $₫$ by first rotating $m$ to $m_{0}, g_{m_{0}, m}^{-1} \triangleright m=m_{0}$, secondly rotating $m_{0}$ as prescribed by $g, g g_{m_{0}, m}^{-1} \triangleright m=g \triangleright m_{0}$, and thirdly undoing the first rotation, $g_{m_{0}, m} g g_{m_{0}, m}^{-1} \triangleright m=g_{m_{0}, m} \triangleright\left(g \triangleright m_{0}\right)$, in other words, by first changing the rotation axis of $g$ such that the new axis stands to the line through the origin and $m$ as the old one stood to the line through the origin and $m_{0}, g_{m_{0}, m} g g_{m_{0}, m}^{-1}$, and secondly rotating $m$ as prescribed by this new rotation.

Let $N_{0}$ be a subset of the sphere $M$, which we think of as a geometrical object on the sphere that has its centre at $m_{0}$, for example, a circle of latitude. The set $N=\left\{g G_{0} \in G / G_{0} \mid g \triangleright m_{0} \in N_{0}\right\}=\left\{G_{m_{0}, m} \mid m \in N_{0}\right\}=\left\{g_{m_{0}, m_{0}} G_{0} \mid\right.$ $\left.m \in N_{0}\right\}$ can be thought of as a realisation of $N_{0}$ in $G / G_{0}$. Indeed, $m_{0} \leqslant N=$ $g_{m_{0}, m_{0}}\left\{g_{m_{0}, m} \mid m \in N_{0}\right\} \triangleright m_{0}=N_{0}$. Furthermore, for each point $m \in M$, the set $m \preccurlyeq N=g_{m_{0}, m} \triangleright N_{0}$ has the same shape and size as $N_{0}$ but its centre at $m$.

Lemma 6. The semi-action $\preccurlyeq i s$

1. transitive, which means that the set $M$ is non-empty and

$$
\forall m \in M \forall m^{\prime} \in M \exists \mathfrak{g} \in G / G_{0}: m \preccurlyeq \mathfrak{g}=m^{\prime} ;
$$

2. free, which means that

$$
\forall \mathfrak{g} \in G / G_{0} \forall \mathfrak{g}^{\prime} \in G / G_{0}:\left(\exists m \in M: m \leqslant \mathfrak{g}=m \preccurlyeq \mathfrak{g}^{\prime}\right) \Longrightarrow \mathfrak{g}=\mathfrak{g}^{\prime} .
$$

Proof. 1. Let $m \in M$ and let $m^{\prime} \in M$. Put $m^{\prime \prime}=g_{m_{0}, m}^{-1} \triangleright m^{\prime}$. Because $\triangleright$ is transitive, there is a $g \in G$ such that $g \triangleright m_{0}=m^{\prime \prime}$. Hence,

$$
\begin{aligned}
m \star g G_{0} & =g_{m_{0}, m} g \triangleright m_{0} \\
& =g_{m_{0}, m} \triangleright\left(g \triangleright m_{0}\right) \\
& =g_{m_{0}, m} \triangleright m^{\prime \prime} \\
& =g_{m_{0}, m} \triangleright\left(g_{m_{0}, m}^{-1} \triangleright m^{\prime}\right) \\
& =e_{G} \triangleright m^{\prime} \\
& =m^{\prime} .
\end{aligned}
$$

2. Let $g G_{0}$ and $g^{\prime} G_{0}$ be two elements of $G / G_{0}$, and let $m$ be an element of $M$ such that $m \leqslant g G_{0}=m \leqslant g^{\prime} G_{0}$. Then, $g_{m_{0}, m} g \triangleright m_{0}=g_{m_{0}, m} g^{\prime} \triangleright m_{0}$. Hence, $g \triangleright m_{0}=g^{\prime} \triangleright m_{0}$. Therefore, $g^{-1} g^{\prime} \triangleright m_{0}=m_{0}$. Thus, $g^{-1} g^{\prime} \in G_{0}$. In conclusion, $g G_{0}=g^{\prime} G_{0}$. 
Lemma 7. The semi-action $₫$

1. semi-commutes with $\triangleright$, which means that, for each subgroup $H$ of $G$ such that $\left\{g_{m_{0}, m} \mid m \in M\right\} \subseteq H$,

$\forall m \in M \forall h \in H \exists h_{0} \in H_{0}: \forall \mathfrak{g} \in G / G_{0}:(h \triangleright m) \Vdash \mathfrak{g}=h \triangleright\left(m \preccurlyeq h_{0} \cdot \mathfrak{g}\right) ;$

2. exhausts its defect with respect to its semi-commutativity with $\triangleright$ in $m_{0}$, which means that, for each subgroup $H$ of $G$ such that $\left\{g_{m_{0}, m} \mid m \in M\right\} \subseteq$ $H$,

$$
\forall h_{0} \in H_{0} \forall \mathfrak{g} \in G / G_{0}:\left(h_{0}^{-1} \triangleright m_{0}\right) \preccurlyeq \mathfrak{g}=h_{0}^{-1} \triangleright\left(m_{0} \preccurlyeq h_{0} \cdot \mathfrak{g}\right) .
$$

Proof. Let $H$ be a subgroup of $G$ such that $\left\{g_{m_{0}, m} \mid m \in M\right\} \subseteq H$.

1. Let $h \in H$ and let $m \in M$. Put $h_{0}=g_{m_{0}, m}^{-1} h^{-1} g_{m_{0}, h \triangleright m}$. Because $g_{m_{0}, m}^{-1} \in H$, $g_{m_{0}, h \triangleright m}^{-1} \in H$, and

$$
\begin{aligned}
g_{m_{0}, m}^{-1} h^{-1} g_{m_{0}, h \triangleright m} \triangleright m_{0} & =g_{m_{0}, m}^{-1} h^{-1} \triangleright(h \triangleright m) \\
& =g_{m_{0}, m}^{-1} \triangleright m \\
& =m_{0},
\end{aligned}
$$

we have $h_{0} \in H_{0}$. Moreover, for each $g G_{0} \in G / G_{0}$,

$$
\begin{aligned}
(h \triangleright m) \preccurlyeq g G_{0} & =g_{m_{0}, h \triangleright m} g \triangleright m_{0} \\
& =h g_{m_{0}, m} h_{0} g \triangleright m_{0} \\
& =h \triangleright\left(g_{m_{0}, m} h_{0} g \triangleright m_{0}\right) \\
& =h \triangleright\left(m \preccurlyeq h_{0} \cdot g G_{0}\right) .
\end{aligned}
$$

2. For each $h_{0} \in H_{0}$ and each $g G_{0} \in G / G_{0}$, because $g_{m_{0}, m_{0}}=e_{G}$,

$$
\begin{aligned}
\left(h_{0}^{-1} \triangleright m_{0}\right) \preccurlyeq g G_{0} & =m_{0} \preccurlyeq g G_{0} \\
& =g_{m_{0}, m_{0}} g \triangleright m_{0} \\
& =h_{0}^{-1} g_{m_{0}, m_{0}} h_{0} g \triangleright m_{0} \\
& =h_{0}^{-1} \triangleright\left(g_{m_{0}, m_{0}} h_{0} g \triangleright m_{0}\right) \\
& =h_{0}^{-1} \triangleright\left(m_{0} \preccurlyeq h_{0} g G_{0}\right) \\
& =h_{0}^{-1} \triangleright\left(m_{0} \preccurlyeq h_{0} \cdot g G_{0}\right) .
\end{aligned}
$$

Lemma 8. The maps

$$
\left\{\begin{aligned}
m_{0} \star_{-}: G / G_{0} & \rightarrow M, \\
\mathfrak{g} & \mapsto m_{0} \triangleleft \mathfrak{g},
\end{aligned}\right\} \text { and }\left\{\begin{aligned}
\iota: M & \rightarrow G / G_{0}, \\
m & \mapsto G_{m_{0}, m},
\end{aligned}\right\}
$$

are inverse to each other and, under the identification of $G / G_{0}$ with $M$ by either of these maps,

$$
\forall m \in M \forall \mathfrak{g} \in G / G_{0} \simeq M: m \preccurlyeq \mathfrak{g}=g_{m_{0}, m} \triangleright \mathfrak{g} .
$$


Proof. According to Lemma 4, the map $\iota$ is bijective and, for each $m \in M$,

$$
\begin{aligned}
m_{0} \preccurlyeq \iota(m) & =m_{0} \Vdash G_{m_{0}, m} \\
& =m_{0} \Vdash g_{m_{0}, m} G_{0} \\
& =g_{m_{0}, m_{0}} g_{m_{0}, m} \triangleright m_{0} \\
& =e_{G} \triangleright m \\
& =m .
\end{aligned}
$$

Therefore, $m_{0}{ }_{-}=\iota^{-1}$. Moreover, for each $g G_{0} \in G / G_{0}$,

$$
\begin{aligned}
m \varangle g G_{0} & =g_{m_{0}, m} g \triangleright m_{0} \\
& =g_{m_{0}, m} \triangleright\left(g \triangleright m_{0}\right) \\
& =g_{m_{0}, m} \triangleright \iota^{-1}\left(G_{m_{0}, g \triangleright m_{0}}\right) \\
& =g_{m_{0}, m} \triangleright \iota^{-1}\left(g G_{0}\right) .
\end{aligned}
$$

\section{Semi-Cellular and Cellular Automata}

In this section, let $\mathcal{R}=(\mathcal{M}, \mathcal{K})=\left((M, G, \triangleright),\left(m_{0},\left\{g_{m_{0}, m}\right\}_{m \in M}\right)\right)$ be a cell space.

Definition 10. Let $Q$ be a set, let $N$ be a subset of $G / G_{0}$ such that $G_{0} \cdot N \subseteq N$, and let $\delta$ be a map from $Q^{N}$ to $Q$. The quadruple $\mathcal{C}=(\mathcal{R}, Q, N, \delta)$ is called semi-cellular automaton, each element $q \in Q$ is called state, the set $N$ is called neighbourhood, each element $n \in N$ is called neighbour, and the map $\delta$ is called local transition function.

Example 11. In the situation of Example 9, the semi-cellular automata over $\mathcal{R}$ are the usual cellular automata over the group $G$.

Example 12. In the situation of Example 10, let $Q$ be the set $\{0,1\}$, let $N_{0}$ be the union of all circles of latitude between $45^{\circ}$ and $90^{\circ}$ north, which is a curved circular disk of radius $\pi / 4$ with the north pole $m_{0}$ at its centre, let $N$ be the set $\left\{g G_{0} \mid g \triangleright m_{0} \in N_{0}\right\}$, and let

$$
\delta: Q^{N} \rightarrow Q, \ell \mapsto \begin{cases}0, & \text { if } \forall n \in N: \ell(n)=0, \\ 1, & \text { if } \exists n \in N: \ell(n)=1 .\end{cases}
$$

The quadruple $\mathcal{C}=(\mathcal{R}, Q, N, \delta)$ is a semi-cellular automaton.

In the remainder of this section, let $\mathcal{C}=(\mathcal{R}, Q, N, \delta)$ be a semi-cellular automaton.

Definition 11. Let $E$ be a subset of $N$. It is called essential neighbourhood if and only if

$$
\forall \ell \in Q^{N} \forall \ell^{\prime} \in Q^{N}: \ell \uparrow_{E}=\ell^{\prime} \uparrow_{E} \Longrightarrow \delta(\ell)=\delta\left(\ell^{\prime}\right) .
$$


Definition 12. Each map $\ell \in Q^{N}$ is called local configuration. The stabiliser $G_{0}$ acts on $Q^{N}$ on the left by

$$
\begin{aligned}
\bullet: G_{0} \times Q^{N} & \rightarrow Q^{N}, \\
\left(g_{0}, \ell\right) & \mapsto\left[n \mapsto \ell\left(g_{0}^{-1} \cdot n\right)\right] .
\end{aligned}
$$

Definition 13. The semi-cellular automaton $\mathcal{C}$ is called cellular automaton if and only if its local transition function $\delta$ is •-invariant.

Example 13. In the situation of Example 11, the stabiliser $G_{0}$ of the neutral element $m_{0}$ is the trivial subgroup $\left\{e_{G}\right\}$ of $G$. Therefore, for each semi-cellular automaton over $\mathcal{R}$, its local transition function is •-invariant and hence it is a cellular automaton.

Example 14. In the situation of Example 12, think of 0 as black, 1 as white, and of local configurations as black-and-white patterns on $N_{0}=m_{0} \leqslant N$. The rotations $G_{0}$ about the $z$-axis act on these patterns by $\bullet$ by rotating them. The local transition function $\delta$ maps the black pattern to 0 and all others to 1 , which is invariant under rotations. Therefore, the quadruple $\mathcal{C}$ is a cellular automaton.

Definition 14. Each map $c \in Q^{M}$ is called global configuration. The group $G$ acts on $Q^{M}$ on the left by

$$
\begin{aligned}
\triangleright: G \times Q^{M} & \rightarrow Q^{M}, \\
(g, c) & \mapsto\left[m \mapsto c\left(g^{-1} \triangleright m\right)\right] .
\end{aligned}
$$

Definition 15. For each global configuration $c \in Q^{M}$ and each cell $m \in M$, the local configuration

$$
\begin{aligned}
N & \rightarrow Q, \\
n & \mapsto c(m \leqslant n),
\end{aligned}
$$

is called observed by $m$ in $c$.

Remark 1. Because the semi-action $\varangle$ is free, for each local configuration $\ell \in Q^{N}$ and each cell $m \in M$, there is a global configuration $c \in Q^{M}$ such that the local configuration observed by $m$ in $c$ is $\ell$.

Definition 16. The map

$$
\begin{aligned}
\Delta: Q^{M} & \rightarrow Q^{M}, \\
c & \mapsto[m \mapsto \delta(n \mapsto c(m \preccurlyeq n))],
\end{aligned}
$$

is called global transition function.

Example 15. In the situation of Example 14, repeated applications of the global transition function of $\mathcal{C}$ grows white regions on $M$. 
Remark 2. For each subset $A$ of $M$ and each global configuration $c \in Q^{M}$, the states of the cells $A$ in $\Delta(c)$ depends at most on the states of the cells $A \leqslant N$ in c. More precisely,

$$
\forall A \subseteq M \forall c \in Q^{M} \forall c^{\prime} \in Q^{M}: c \uparrow_{A \preccurlyeq N}=c^{\prime} \uparrow_{A \preccurlyeq N} \Longrightarrow \Delta(c) \uparrow_{A}=\Delta\left(c^{\prime}\right) \uparrow_{A} .
$$

Lemma 9. Let $m$ be an element of $M$, let $g$ be an element of $G$, and let $g_{0}$ be an element of $G_{0}$ such that

$$
\forall n \in N:\left(g^{-1} \triangleright m\right) \preccurlyeq n=g^{-1} \triangleright\left(m \leqslant g_{0} \cdot n\right) .
$$

For each global configuration $c \in Q^{M}$,

$$
\left[n \mapsto c\left(\left(g^{-1} \triangleright m\right) \preccurlyeq n\right)\right]=g_{0}^{-1} \bullet[n \mapsto(g \triangleright c)(m \preccurlyeq n)] .
$$

Proof. For each global configuration $c \in Q^{M}$,

$$
\begin{aligned}
{\left[n \mapsto c\left(\left(g^{-1} \triangleright m\right) \preccurlyeq n\right)\right] } & =\left[n \mapsto c\left(g^{-1} \triangleright\left(m \preccurlyeq g_{0} \cdot n\right)\right)\right] \\
& =g_{0}^{-1} \bullet\left[n \mapsto c\left(g^{-1} \triangleright(m \preccurlyeq n)\right)\right] \\
& =g_{0}^{-1} \bullet[n \mapsto(g \triangleright c)(m \preccurlyeq n)] .
\end{aligned}
$$

Definition 17. The set $N_{0}=m_{0} \triangleleft N$ is called neighbourhood of $m_{0}$.

Definition 18. The map

$$
\begin{aligned}
\delta_{0}: Q^{N_{0}} & \rightarrow Q, \\
\ell_{0} & \mapsto \delta\left(n \mapsto \ell_{0}\left(m_{0} \preccurlyeq n\right)\right),
\end{aligned}
$$

is called local transition function of $m_{0}$.

Lemma 10. The global transition function $\Delta$ of $\mathcal{C}$ is identical to the map

$$
\begin{aligned}
\Delta_{0}: Q^{M} & \rightarrow Q^{M}, \\
c & \mapsto\left[m \mapsto \delta_{0}\left(\left(g_{m_{0}, m}^{-1} \triangleright c\right)\left\lceil_{N_{0}}\right)\right] .\right.
\end{aligned}
$$

Proof. Let $c \in Q^{M}$ and let $m \in M$. For each $n=g G_{0} \in N$,

$$
\begin{aligned}
m \star n & =g_{m_{0}, m} g \triangleright m_{0} \\
& =g_{m_{0}, m} g_{m_{0}, m_{0}} g \triangleright m_{0} \\
& =g_{m_{0}, m} \triangleright\left(g_{m_{0}, m_{0}} g \triangleright m_{0}\right) \\
& =g_{m_{0}, m} \triangleright\left(m_{0} \preccurlyeq n\right)
\end{aligned}
$$

and thus

$$
c(m \preccurlyeq n)=c\left(g_{m_{0}, m} \triangleright\left(m_{0} \preccurlyeq n\right)\right)=\left(g_{m_{0}, m}^{-1} \triangleright c\right)\left(m_{0} \preccurlyeq n\right) .
$$

Therefore,

$$
\Delta(c)(m)=\delta(n \mapsto c(m \preccurlyeq n))
$$




$$
\begin{aligned}
& =\delta\left(n \mapsto\left(g_{m_{0}, m}^{-1} \triangleright c\right)\left(m_{0} \leqslant n\right)\right) \\
& =\delta_{0}\left(n_{0} \mapsto\left(g_{m_{0}, m}^{-1} \triangleright c\right)\left(n_{0}\right)\right) \\
& =\delta_{0}\left(\left(g_{m_{0}, m}^{-1} \triangleright c\right) \uparrow_{N_{0}}\right) \\
& =\Delta_{0}(c)(m) .
\end{aligned}
$$

In conclusion, $\Delta=\Delta_{0}$.

\section{Invariance, Equivariance, Determination, and Composition of Global Transition Functions}

In Theorem 1 we show that a global transition function does not depend on the choice of coordinate system. In Theorem 2 we show that a global transition function is -equivariant if and only if the local transition function is $\bullet$-invariant. In Theorem 3 we show that a global transition function is determined by its behaviour in the origin. And in Theorem 4 we show that the composition of two global transition functions is a global transition function.

Lemma 11. Let $\triangleright$ be a left group action of $G$ on $M$. The group $G$ acts on $\bigcup_{m \in M} G / G_{m}$ on the left by

$$
\begin{aligned}
\circ: G \times \bigcup_{m \in M} G / G_{m} & \rightarrow \bigcup_{m \in M} G / G_{m}, \\
\left(g, g^{\prime} G_{m}\right) & \mapsto g g^{\prime} G_{m} g^{-1}\left(=g g^{\prime} g^{-1} G_{g \triangleright m}\right),
\end{aligned}
$$

such that, for each element $g \in G$ and each element $m \in M$, the map

$$
\begin{aligned}
\left(g \circ{ }_{-}\right) \Gamma_{G / G_{m} \rightarrow G / G_{g \triangleright m}: G / G_{m}} & \rightarrow G / G_{g \triangleright m}, \\
g^{\prime} G_{m} & \mapsto g \circ g^{\prime} G_{m},
\end{aligned}
$$

is bijective.

Proof. To see that the maps $\circ$ and $\left(g \circ{ }_{-}\right) \Gamma_{G / G_{m} \rightarrow G / G_{g \triangleright m}}$ are well-defined, let $g \in G$, let $m \in M$, let $g^{\prime} G_{m} \in G / G_{m}$, and put $m^{\prime} \stackrel{g \triangleright m}{=} g \triangleright m$. According to Lemma 1, we have $G_{m^{\prime}}=g G_{m} g^{-1}$. Therefore

$$
g g^{\prime} G_{m} g^{-1}=g g^{\prime}\left(g^{-1} g\right) G_{m} g^{-1}=\left(g g^{\prime} g^{-1}\right)\left(g G_{m} g^{-1}\right)=g g^{\prime} g^{-1} G_{m^{\prime}} \in G / G_{m^{\prime}} .
$$

To show that the map $\circ$ is a left group action, let $g G_{m} \in \bigcup_{m \in M} G / G_{m}$. We have $e_{G} \circ g G_{m}=g G_{m}$. Moreover, for each $g^{\prime} \in G$ and each $g^{\prime \prime} \in G$,

$$
g^{\prime} g^{\prime \prime} \circ g G_{m}=g^{\prime} g^{\prime \prime} g G_{m}\left(g^{\prime \prime}\right)^{-1}\left(g^{\prime}\right)^{-1}=g^{\prime} \circ g^{\prime \prime} g G_{m}\left(g^{\prime \prime}\right)^{-1}=g^{\prime} \circ\left(g^{\prime \prime} \circ g G_{m}\right) .
$$

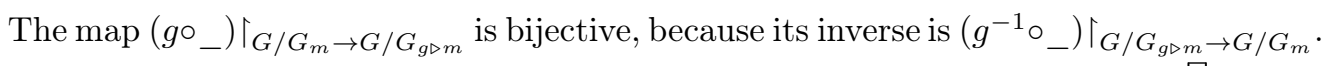


Lemma 12. Let $\mathcal{M}=(M, G, \triangleright)$ be a left homogeneous space, let $\mathcal{K}=\left(m_{0}\right.$, $\left.\left\{g_{m_{0}, m}\right\}_{m \in M}\right)$ and $\mathcal{K}^{\prime}=\left(m_{0}^{\prime},\left\{g_{m_{0}^{\prime}, m}^{\prime}\right\}_{m \in M}\right)$ be two coordinate systems for $\mathcal{M}$, let $H$ be a subgroup of $G$ such that $\left\{g_{m_{0}, m} \mid m \in M\right\} \cup\left\{g_{m_{0}^{\prime}, m}^{\prime} \mid m \in M\right\} \subseteq H$, and let $h$ be an element of $H$ such that $h \triangleright m_{0}=m_{0}^{\prime}$. Then,

$$
\forall m \in M \exists h_{0} \in H_{0}: \forall \mathfrak{g}^{\prime} \in G / G_{0}^{\prime}: m \S^{\prime} \mathfrak{g}^{\prime}=m \preccurlyeq h_{0} \cdot\left(h^{-1} \circ \mathfrak{g}^{\prime}\right) .
$$

Proof. Let $m \in M$. Put $h_{0}=g_{m_{0}, m}^{-1} g_{m_{0}^{\prime}, m}^{\prime} h$. Then, $h_{0} \in H_{0}$ and $g_{m_{0}^{\prime}, m}^{\prime}=$ $g_{m_{0}, m} h_{0} h^{-1}$. Furthermore, let $g G_{0}^{\prime} \in G / G_{0}^{\prime}$. Then,

$$
\begin{aligned}
m \S^{\prime} g G_{0}^{\prime} & =g_{m_{0}^{\prime}, m}^{\prime} g\left(g_{m_{0}^{\prime}, m}^{\prime}\right)^{-1} \triangleright m \\
& =g_{m_{0}, m} h_{0} h^{-1} g h h_{0}^{-1} g_{m_{0}, m}^{-1} \triangleright m \\
& =m \leqslant h_{0} h^{-1} g h h_{0}^{-1} G_{0} .
\end{aligned}
$$

Thus, because $h_{0}^{-1} G_{0}=G_{0}$ and $h G_{0} h^{-1}=h \circ G_{0}=G_{0}^{\prime}$,

$$
\begin{aligned}
m s^{\prime} g G_{0}^{\prime} & =m \preccurlyeq h_{0} \cdot h^{-1} g h G_{0} \\
& =m \preccurlyeq h_{0} \cdot h^{-1} g h G_{0} h^{-1} h \\
& =m \preccurlyeq h_{0} \cdot h^{-1} g G_{0}^{\prime} h \\
& =m \preccurlyeq h_{0} \cdot\left(h^{-1} \circ g G_{0}^{\prime}\right) .
\end{aligned}
$$

Theorem 1. In the situation of Lemma[12, let $\mathcal{C}=((\mathcal{M}, \mathcal{K}), Q, N, \delta)$ be a semicellular automaton such that $\delta$ is $\bullet_{H_{0}}$-invariant, let $N^{\prime}$ be the set $h \circ N$, and let

$$
\begin{aligned}
\delta^{\prime}: Q^{N^{\prime}} & \rightarrow Q, \\
\ell^{\prime} & \mapsto \delta\left(n \mapsto \ell^{\prime}(h \circ n)\right) .
\end{aligned}
$$

The quadruple $\left(\left(\mathcal{M}, \mathcal{K}^{\prime}\right), Q, N^{\prime}, \delta^{\prime}\right)$ is a semi-cellular automaton whose global transition function is identical to the one of $\mathcal{C}$.

Proof. We have $N^{\prime} \subseteq G / G_{0}^{\prime}$ and $G_{0}^{\prime} \cdot N^{\prime} \subseteq N^{\prime}$. Moreover, let $c \in Q^{M}$ and let $m \in M$. According to Lemma 12, there is an $h_{0} \in H_{0}$ such that

$$
\forall n^{\prime} \in N^{\prime}: m \varangle^{\prime} n^{\prime}=m \varangle h_{0} \cdot\left(h^{-1} \circ n^{\prime}\right) .
$$

Therefore, because $\delta$ is $\bullet_{H_{0}}$-invariant,

$$
\begin{aligned}
\Delta^{\prime}(c)(m) & =\delta^{\prime}\left(n^{\prime} \mapsto c\left(m \varangle^{\prime} n^{\prime}\right)\right) \\
& =\delta^{\prime}\left(n^{\prime} \mapsto c\left(m \varangle h_{0} \cdot\left(h^{-1} \circ n^{\prime}\right)\right)\right) \\
& =\delta\left(n \mapsto c\left(m \varangle h_{0} \cdot\left(h^{-1} \circ(h \circ n)\right)\right)\right) \\
& =\delta\left(n \mapsto c\left(m \preccurlyeq h_{0} \cdot n\right)\right) \\
& =\delta\left(h_{0}^{-1} \bullet[n \mapsto c(m \preccurlyeq n)]\right) \\
& =\delta(n \mapsto c(m \leqslant n)) \\
& =\Delta(c)(m) .
\end{aligned}
$$

In conclusion, $\Delta^{\prime}=\Delta$. 
Corollary 1. Let $(\mathcal{M}, \mathcal{K})=\left((M, G, \triangleright),\left(m_{0},\left\{g_{m_{0}, m}\right\}_{m \in M}\right)\right)$ be a cell space and let $\mathcal{C}=((\mathcal{M}, \mathcal{K}), Q, N, \delta)$ be a cellular automaton. For each coordinate system $\mathcal{K}^{\prime}=\left(m_{0}^{\prime},\left\{g_{m_{0}^{\prime}, m}^{\prime}\right\}_{m \in M}\right)$ for $\mathcal{M}$, there is a cellular automaton $\left(\left(\mathcal{M}, \mathcal{K}^{\prime}\right), Q, N^{\prime}\right.$, $\left.\delta^{\prime}\right)$ whose global transition function is identical to the one of $\mathcal{C}$.

Proof. This follows directly from Theorem 1 with $H=G$ and $h=g_{m_{0}, m_{0}^{\prime}}$.

Theorem 2. Let $\mathcal{R}=\left((M, G, \triangleright),\left(m_{0},\left\{g_{m_{0}, m}\right\}_{m \in M}\right)\right)$ be a cell space, let $\mathcal{C}=$ $(\mathcal{R}, Q, N, \delta)$ be a semi-cellular automaton, and let $H$ be a subgroup of $G$ such that $\left\{g_{m_{0}, m} \mid m \in M\right\} \subseteq H$.

1. If the local transition function $\delta$ is $\bullet_{H_{0}}$-invariant, then the global transition function $\Delta$ is ${ }_{H}$-equivariant.

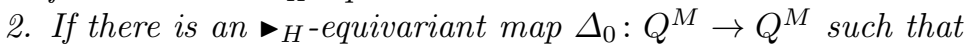

$$
\forall c \in Q^{M}: \Delta_{0}(c)\left(m_{0}\right)=\delta\left(n \mapsto c\left(m_{0} \preccurlyeq n\right)\right),
$$

then the local transition function $\delta$ is $\bullet_{H_{0}}$-invariant.

3. The local transition function $\delta$ is $\bullet_{H_{0}}$-invariant if and only if the global transition function $\Delta$ is $\boldsymbol{H}_{\text {-equivariant. }}$

Proof. 1. Let $\delta$ be $\bullet_{H_{0}}$-invariant. Furthermore, let $h \in H$, let $c \in Q^{M}$, and let $m \in M$. According to Item 1 of Lemma 7 , there is an $h_{0} \in H_{0}$ such that

$$
\forall n \in N:\left(h^{-1} \triangleright m\right) \preccurlyeq n=h^{-1} \triangleright\left(m \leqslant h_{0} \cdot n\right) .
$$

Hence, according to Lemma 9 ,

$$
\begin{aligned}
(h \triangleright \Delta(c))(m) & =\Delta(c)\left(h^{-1} \triangleright m\right) \\
& =\delta\left(n \mapsto c\left(\left(h^{-1} \triangleright m\right) \preccurlyeq n\right)\right) \\
& =\delta\left(h_{0}^{-1} \bullet[n \mapsto(h \triangleright c)(m \preccurlyeq n)]\right) \\
& =\delta(n \mapsto(h \triangleright c)(m \preccurlyeq n)) \\
& =\Delta(h \triangleright c)(m) .
\end{aligned}
$$

In conclusion, $\Delta$ is $\boldsymbol{H}^{\text {-equivariant. }}$

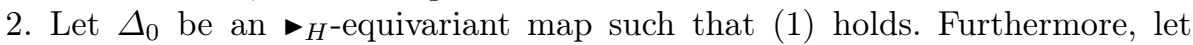
$\ell \in Q^{N}$ and let $h_{0} \in H_{0}$. According to Remark 1, there is a $c \in Q^{M}$ such that $\left[n \mapsto c\left(m_{0} \preccurlyeq n\right)\right]=\ell$. According to Item 2 of Lemma 7 ,

$$
\forall n \in N:\left(h_{0}^{-1} \triangleright m\right) \preccurlyeq n=h_{0}^{-1} \triangleright\left(m \preccurlyeq h_{0} \cdot n\right) .
$$

Hence, according to Lemma 9 ,

$$
\begin{aligned}
\delta\left(h_{0} \bullet \ell\right) & =\delta\left(h_{0} \bullet\left[n \mapsto c\left(m_{0} \preccurlyeq n\right)\right]\right) \\
& =\delta\left(h_{0} \bullet\left[n \mapsto c\left(\left(h_{0}^{-1} \triangleright m_{0}\right) \preccurlyeq n\right)\right]\right) \\
& =\delta\left(n \mapsto\left(h_{0} \triangleright c\right)\left(m_{0} \preccurlyeq n\right)\right) \\
& =\Delta_{0}\left(h_{0} \bullet c\right)\left(m_{0}\right) .
\end{aligned}
$$


Because $\Delta_{0}$ is ${ }_{H}$-equivariant,

$$
\begin{aligned}
\Delta_{0}\left(h_{0} \triangleright c\right)\left(m_{0}\right) & =\left(h_{0} \triangleright \Delta_{0}(c)\right)\left(m_{0}\right) \\
& =\Delta_{0}(c)\left(h_{0}^{-1} \triangleright m_{0}\right) \\
& =\Delta_{0}(c)\left(m_{0}\right) \\
& =\delta(\ell) .
\end{aligned}
$$

Put the last two chains of equalities together to see that $\delta\left(h_{0} \bullet \ell\right)=\delta(\ell)$. In conclusion, $\delta$ is $\bullet_{H_{0}}$-invariant.

3. If $\delta$ is $\bullet H_{0}$-invariant, then $\Delta$ is $\bullet_{H}$-equivariant by Item 1 , On the other hand,

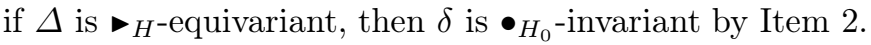

Corollary 2. Let $\mathcal{C}$ be a semi-cellular automaton. It is a cellular automaton if and only if its global transition function is $>$-equivariant.

Proof. This follows directly from Item 3 of Theorem 2 with $H=G$.

Theorem 3. Let $\mathcal{R}=\left((M, G, \triangleright),\left(m_{0},\left\{g_{m_{0}, m}\right\}_{m \in M}\right)\right)$ be a cell space, let $\mathcal{C}=$ $(\mathcal{R}, Q, N, \delta)$ be a semi-cellular automaton, let $\Delta_{0}$ be a map from $Q^{M}$ to $Q^{M}$, and let $H$ be a subgroup of $G$ such that $\left\{g_{m_{0}, m} \mid m \in M\right\} \subseteq H$. The following statements are equivalent:

1. The local transition function $\delta$ is $\bullet_{H_{0}}$-invariant and the global transition function of $\mathcal{C}$ is $\Delta_{0}$;

2. The global transition function $\Delta_{0}$ is $\boldsymbol{H}_{\text {-equivariant and }}$

$$
\forall c \in Q^{M}: \Delta_{0}(c)\left(m_{0}\right)=\delta\left(n \mapsto c\left(m_{0} \preccurlyeq n\right)\right) .
$$

Proof. First, let $\delta$ be $\bullet_{H_{0}}$-invariant and let $\Delta_{0}$ be the global transition function of $\mathcal{C}$. According to Item 1 of Theorem 2 the map $\Delta_{0}$ is $\boldsymbol{}_{H}$-equivariant and, according to Definition 16, (2) holds.

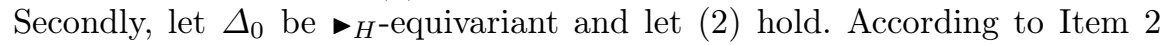
of Theorem 2, the local transition function $\delta$ is $\bullet_{H_{0}}$-invariant. Furthermore, let $c \in Q^{M}$ and let $m \in M$. Put $h=g_{m_{0}, m}^{-1} \in H$. Then,

$$
\Delta_{0}(c)(m)=\Delta_{0}(c)\left(h^{-1} \triangleright m_{0}\right)=\left(h \triangleright \Delta_{0}(c)\right)\left(m_{0}\right) .
$$

Because $\Delta_{0}$ is $H_{\text {-equivariant, }}$

$$
\left(h \triangleright \Delta_{0}(c)\right)\left(m_{0}\right)=\Delta_{0}(h \triangleright c)\left(m_{0}\right)=\delta\left(n \mapsto(h \triangleright c)\left(m_{0} \preccurlyeq n\right)\right) .
$$

According to Lemma 7 there is an $h_{0} \in H_{0}$ such that, for each $n \in N$, we have $\left(h^{-1} \triangleright m_{0}\right) \preccurlyeq n=h^{-1} \triangleright\left(m_{0} \preccurlyeq h_{0} \cdot n\right)$. Therefore, according to Lemma 9 ,

$$
\begin{aligned}
\delta\left(n \mapsto(h \triangleright c)\left(m_{0} \triangleleft n\right)\right) & =\delta\left(h_{0} \bullet\left[n \mapsto c\left(\left(h^{-1} \triangleright m_{0}\right) \preccurlyeq n\right)\right]\right) \\
& =\delta\left(h_{0} \bullet[n \mapsto c(m \varangle n)]\right) .
\end{aligned}
$$

Because $\delta$ is $\bullet_{H_{0}}$-invariant,

$$
\delta\left(h_{0} \bullet[n \mapsto c(m \preccurlyeq n)]\right)=\delta(n \mapsto c(m \preccurlyeq n)) .
$$

Put the last four chains of equalities together to see that $\Delta_{0}(c)(m)=\delta(n \mapsto$ $c(m \preccurlyeq n))$. In conclusion, $\Delta_{0}$ is the global transition function of $\mathcal{C}$. 
Theorem 4. Let $\mathcal{R}=\left((M, G, \triangleright),\left(m_{0},\left\{g_{m_{0}, m}\right\}_{m \in M}\right)\right)$ be a cell space, let $\mathcal{C}=$ $(\mathcal{R}, Q, N, \delta)$ and $\mathcal{C}^{\prime}=\left(\mathcal{R}, Q, N^{\prime}, \delta^{\prime}\right)$ be two semi-cellular automata, and let $H$ be a subgroup of $G$ such that $\left\{g_{m_{0}, m} \mid m \in M\right\} \subseteq H$, and $\delta$ and $\delta^{\prime}$ are $\bullet_{H_{0}}$-invariant. Furthermore, let

$$
N^{\prime \prime}=\left\{g \cdot n^{\prime} \mid n \in N, n^{\prime} \in N^{\prime}, g \in n\right\}
$$

and let

$$
\begin{aligned}
\delta^{\prime \prime}: Q^{N^{\prime \prime}} & \rightarrow Q, \\
\ell^{\prime \prime} & \mapsto \delta\left(n \mapsto \delta^{\prime}\left(n^{\prime} \mapsto \ell^{\prime \prime}\left(g_{m_{0}, m_{0} \preccurlyeq n} \cdot n^{\prime}\right)\right)\right) .
\end{aligned}
$$

The quadruple $\mathcal{C}^{\prime \prime}=\left(\mathcal{R}, Q, N^{\prime \prime}, \delta^{\prime \prime}\right)$ is a semi-cellular automaton whose local transition function is $\boldsymbol{H}_{0}$-invariant and whose global transition function is $\Delta \circ$ $\Delta^{\prime}$.

Proof. The quadruple $\mathcal{C}^{\prime \prime}=\left(\mathcal{R}, Q, N^{\prime \prime}, \delta^{\prime \prime}\right)$ is a semi-cellular automaton. Because $\delta$ and $\delta^{\prime}$ are $\bullet_{H_{0}}$-invariant, according to Item 10 of Theorem 2, the maps $\Delta$ and $\Delta^{\prime}$ are $H_{H}$-equivariant and hence $\Delta \circ \Delta^{\prime}$ also. Because $g_{m_{0}, m_{0}}=e_{G}$,

$$
\forall n \in N \forall n^{\prime} \in N^{\prime}:\left(m_{0} \preccurlyeq n\right) \preccurlyeq n^{\prime}=m_{0} \preccurlyeq g_{m_{0}, m_{0} \preccurlyeq n} \cdot n^{\prime} .
$$

Thus, for each $c \in Q^{M}$,

$$
\begin{aligned}
\left(\Delta \circ \Delta^{\prime}\right)(c)\left(m_{0}\right) & =\delta\left(n \mapsto \Delta^{\prime}(c)\left(m_{0} \preccurlyeq n\right)\right) \\
& =\delta\left(n \mapsto \delta^{\prime}\left(n^{\prime} \mapsto c\left(\left(m_{0} \preccurlyeq n\right) \preccurlyeq n^{\prime}\right)\right)\right) \\
& =\delta\left(n \mapsto \delta^{\prime}\left(n^{\prime} \mapsto c\left(m_{0} \preccurlyeq g_{m_{0}, m_{0} \preccurlyeq n} \cdot n^{\prime}\right)\right)\right) \\
& =\delta^{\prime \prime}\left(n^{\prime \prime} \mapsto c\left(m_{0} \preccurlyeq n^{\prime \prime}\right)\right) .
\end{aligned}
$$

Therefore, according to Theorem [3, the local transition function $\delta^{\prime \prime}$ is $\bullet_{H_{0}}$ invariant and the global transition function of $\mathcal{C}^{\prime \prime}$ is $\Delta \circ \Delta^{\prime}$.

\section{Curtis-Hedlund-Lyndon Theorems}

In this section we equip the the set of global configurations with a uniform and a topological structure, and prove a uniform and a topological variant of the Curtis-Hedlund-Lyndon theorem. In Main Theorem 5 , the uniform variant, we show that global transition functions are characterised by -equivariance and uniform continuity. And in its Corollary 3 , the topological variant, that under the assumption that the set of states is finite they are characterised by - -equivariance and continuity.

Definition 19. Let $G$ be a group equipped with a topology. It is called topological if and only if the maps

$$
\left\{\begin{aligned}
G \times G & \rightarrow G, \\
\left(g, g^{\prime}\right) & \mapsto g g^{\prime},
\end{aligned}\right\} \text { and }\left\{\begin{aligned}
G & \rightarrow G, \\
g & \mapsto g^{-1},
\end{aligned}\right\}
$$

are continuous, where $G \times G$ is equipped with the product topology. 
Definition 20. Let $M$ be a set, let $\mathfrak{L}$ be a subset of the power set of $M$, and let $T$ be a subset of $M$. The set $T$ is called transversal of $\mathfrak{L}$ if and only if there is a surjective map $f: \mathfrak{L} \rightarrow T$ such that for each set $A \in \mathfrak{L}$ we have $f(A) \in A$.

Definition 21. Let $M$ and $M^{\prime}$ be two topological spaces and let $f$ be a continuous map from $M$ to $M^{\prime}$. The map $f$ is called

1. proper if and only if, for each compact subset $K$ of $M^{\prime}$, its preimage $f^{-1}(K)$ is a compact subset of $M$;

2. semi-proper if and only if, for each compact subset $K$ of $M^{\prime}$, each transversal of $\left\{f^{-1}(k) \mid k \in K\right\}$ is included in a compact subset of $M$.

Definition 22. Let $M$ be a topological space, let $G$ be a topological group, and let $\mathcal{M}=(M, G, \triangleright)$ be a left group set. The group set $\mathcal{M}$ is called

1. topological if and only if the map $\triangleright$ is continuous;

2. proper if and only if it is topological and the so-called action map

$$
\begin{aligned}
\alpha: G \times M & \rightarrow M \times M, \\
(g, m) & \mapsto(g \triangleright m, m),
\end{aligned}
$$

is proper, where $G \times M$ and $M \times M$ are equipped with their respective product topology;

3. semi-proper if and only if it is topological and its action map is semi-proper.

Remark 3. Each proper map is semi-proper and each proper left group set is semi-proper.

Lemma 13. Let $\mathcal{M}=(M, G, \triangleright)$ be a semi-proper left group set, and let $K$ and $K^{\prime}$ be two compact subsets of $M$. Each transversal of $\left\{G_{k^{\prime}, k} \mid\left(k, k^{\prime}\right) \in K \times K^{\prime}\right\}$ is included in a compact subset of $G$.

Proof. According to Tychonoff's theorem and because product and subspace topologies behave well with each other, the set $K \times K^{\prime}$ is a compact subset of $M \times M$. Because the action map $\alpha$ of $\mathcal{M}$ is semi-proper and the preimage of each tuple $\left(k, k^{\prime}\right) \in K \times K^{\prime}$ under $\alpha$ is $G_{k^{\prime}, k} \times\left\{k^{\prime}\right\}$, each transversal of

$$
\left\{G_{k^{\prime}, k} \times\left\{k^{\prime}\right\} \mid\left(k, k^{\prime}\right) \in K \times K^{\prime}\right\}
$$

is included in a compact subset of $G \times M$. Because the canonical projection $\pi$ of $G \times M$ onto $G$ is continuous, each transversal of

$$
\left\{G_{k^{\prime}, k} \mid\left(k, k^{\prime}\right) \in K \times K^{\prime}\right\}
$$

is included in a compact subset of $G$.

Lemma 14. Let $\mathcal{M}=(M, G, \triangleright)$ be a left group set. Equip $M$ and $G$ with their respective discrete topology. The group set $\mathcal{M}$ is semi-proper. 
Proof. The product topology on $G \times M$ and the one on $M \times M$ are discrete. Hence, each subset of $G \times M$ and each of $M \times M$ is open. Thus, the map $\triangleright$ is continuous. Moreover, each subset of $G \times M$ and each of $M \times M$ is compact if and only if it is finite.

Let $K$ be a finite subset of $M \times M$. Because the set $\left\{\alpha^{-1}(k) \mid k \in K\right\}$, where $\alpha$ is the action map of $\mathcal{M}$, is finite, so is each of its transversals. Thus, the map $\alpha$ is semi-proper. In conclusion, the group set $\mathcal{M}$ is semi-proper.

Definition 23. Let $\mathcal{R}=\left((M, G, \triangleright),\left(m_{0},\left\{g_{m_{0}, m}\right\}_{m \in M}\right)\right)$ be a topological or uniform cell space. Equip $G / G_{0}$ with the topology or uniformity induced by $m_{0} \star_{-}$.

Lemma 15. Let $\mathcal{R}=\left((M, G, \triangleright),\left(m_{0},\left\{g_{m_{0}, m}\right\}_{m \in M}\right)\right)$ be a semi-proper cell space, let $K$ be a compact subset of $M$, and let $E$ be a compact subset of $G / G_{0}$. The set $K \preccurlyeq E$ is included in a compact subset of $M$.

Proof. We have

$$
K \preccurlyeq E=\bigcup_{k \in K} g_{m_{0}, k} \triangleright\left(m_{0} \preccurlyeq E\right)=\left\{g_{m_{0}, k} \mid k \in K\right\} \triangleright\left(m_{0} \preccurlyeq E\right) .
$$

Both, the singleton set $\left\{m_{0}\right\}$ and the set $K$ are compact. Hence, according to Lemma 13, the transversal $\left\{g_{m_{0}, k} \mid k \in K\right\}$ of $\left\{G_{m_{0}, k} \mid\left(m_{0}, k\right) \in\left\{m_{0}\right\} \times K\right\}$ is included in a compact subset of $G$. Thus, the product $\left\{g_{m_{0}, k} \mid k \in K\right\} \times\left(m_{0} \preccurlyeq E\right)$ is included in a compact subset of $G \times M$. Therefore, because $\triangleright$ is continuous, the image $\left\{g_{m_{0}, k} \mid k \in K\right\} \triangleright\left(m_{0} \preccurlyeq E\right)$ is included in a compact subset of $M$.

Definition 24. Let $M$ be a topological space and let $Q$ be a set.

1. The topology on $Q^{M}$ that has for a subbase (and base) the sets

$$
\mathfrak{E}(K, b)=\left\{c \in Q^{M} \mid c \uparrow_{K}=b\right\}, \text { for } b \in Q^{K} \text { and } K \subseteq M \text { compact, }
$$

is called topology of discrete convergence on compacta.

2. The uniformity on $Q^{M}$ that has for a subbase (and base) the sets

$$
\mathfrak{E}(K)=\left\{\left(c, c^{\prime}\right) \in Q^{M} \times Q^{M} \mid c \uparrow_{K}=c^{\prime} \uparrow_{K}\right\}, \text { for } K \subseteq M \text { compact, }
$$

is called uniformity of discrete convergence on compacta.

Remark 4. 1. Let $M$ be equipped with the discrete topology. For each subset $A$ of $M$, the set $A$ is compact if and only if it is finite. Therefore, the topology and uniformity of discrete convergence on compacta on $Q^{M}$ are the prodiscrete topology and uniformity on $Q^{M}$ respectively. For definitions of the latter see Sects. 1.2 and 1.9 in [1]. The set $Q^{M}$ equipped wit the prodiscrete topology is Hausdorff (see Proposition 1.2.1 in [1]) and if $Q$ is finite then it is compact (see the first paragraph in Sect. 1.8 in [1]).

2. If the topological space $M$ is compact, the topology and uniformity of discrete convergence on compacta on $Q^{M}$ is the discrete topology and uniformity on $Q^{M}$ respectively. 
3. The topology induced by the uniformity of discrete convergence on compacta on $Q^{M}$ is the topology of discrete convergence on compacta on $Q^{M}$.

Remark 5. Let $\mathcal{R}=\left((M, G, \triangleright),\left(m_{0},\left\{g_{m_{0}, m}\right\}_{m \in M}\right)\right)$ be a cell space and let $Q$ be a finite set. Equip $M$ and $G$ with their respective discrete topologies, and equip $Q^{M}$ with the prodiscrete topology. According to Lemma 14, the cell space $\mathcal{R}$ is semi-proper. Because the topology on $G / G_{0}$ is discrete, each subset $E$ of $G / G_{0}$ is compact if and only if it is finite. Because $Q^{M}$ is compact, each map $\Delta: Q^{M} \rightarrow Q^{M}$ is uniformly continuous if and only if it is continuous. And, because $Q^{M}$ is Hausdorff, each map $\Delta: Q^{M} \rightarrow Q^{M}$, is a uniform isomorphism if and only if it is continuous and bijective.

Main Theorem 5 (Uniform Variant; Morton Landers Curtis, Gustav Arnold Hedlund, and Roger Conant Lyndon, 1969). Let $\mathcal{R}=((M, G, \triangleright)$, $\left.\left(m_{0},\left\{g_{m_{0}, m}\right\}_{m \in M}\right)\right)$ be a semi-proper cell space, let $Q$ be a set, let $\Delta$ be a map from $Q^{M}$ to $Q^{M}$, let $Q^{M}$ be equipped with the uniformity of discrete convergence on compacta, and let $H$ be a subgroup of $G$ such that $\left\{g_{m_{0}, m} \mid m \in M\right\} \subseteq H$. The following statements are equivalent:

1. The map $\Delta$ is the global transition function of a semi-cellular automaton with - $H_{0}$-invariant local transition function and compact essential neighbourhood.

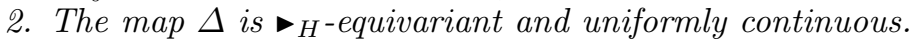

Proof. First, let $\Delta$ be the global transition function of a semi-cellular automaton $\mathcal{C}=(\mathcal{R}, Q, N, \delta)$ such that $\delta$ is $\bullet_{H_{0}}$-invariant and such that there is a compact essential neighbourhood $E$ of $\mathcal{C}$. Then, according to Item 3 of Theorem 2 the

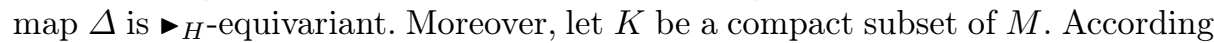
to Lemma 15, the set $K \leqslant E$ is included in a compact subset $L$ of $M$. For each $c \in Q^{M}$ and each $c^{\prime} \in Q^{M}$, if $c \uparrow_{K \diamond E}=c^{\prime} \uparrow_{K \triangleleft E}$, then $\left.\Delta(c)\right|_{K}=\Delta\left(c^{\prime}\right) \uparrow_{K}$, in particular, if $\left.c\right|_{L}=\left.c^{\prime}\right|_{L}$, then $\left.\Delta(c)\right|_{K}=\left.\Delta\left(c^{\prime}\right)\right|_{K}$. Thus,

$$
(\Delta \times \Delta)(\mathfrak{E}(L)) \subseteq \mathfrak{E}(K) .
$$

Because the sets $\mathfrak{E}(K)$, for $K \subseteq M$ compact, constitute a base of the uniformity on $Q^{M}$, the global transition function $\Delta$ is uniformly continuous.

Secondly, let $\Delta$ be as in Item 2 Because $\Delta$ is uniformly continuous, there is a compact subset $E_{0}$ of $M$ such that

$$
(\Delta \times \Delta)\left(\mathfrak{E}\left(E_{0}\right)\right) \subseteq \mathfrak{E}\left(\left\{m_{0}\right\}\right) .
$$

Therefore, for each $c \in Q^{M}$, the state $\Delta(c)\left(m_{0}\right)$ depends at most on $c \uparrow_{E_{0}}$. The subset $E=\left(m_{0} \varangle_{-}\right)^{-1}\left(E_{0}\right)=\left\{G_{m_{0}, m} \mid m \in E_{0}\right\}$ of $G / G_{0}$ is compact. Let $N$ be the set $G_{0} \cdot E$. Then, $G_{0} \cdot N \subseteq N$. And, because $E_{0} \subseteq m_{0} \preccurlyeq N$, for each $c \in Q^{M}$, the state $\Delta(c)\left(m_{0}\right)$ depends at most on $\left.c\right|_{m_{0} \leqslant N}$. Hence, there is a map $\delta: Q^{N} \rightarrow Q$ such that

$$
\forall c \in Q^{M}: \Delta(c)\left(m_{0}\right)=\delta\left(n \mapsto c\left(m_{0} \preccurlyeq n\right)\right) .
$$

The quadruple $\mathcal{C}=(\mathcal{R}, Q, N, \delta)$ is a semi-cellular automaton. Conclude with Theorem 3 that $\delta$ is $\bullet_{H_{0}}$-invariant and that $\Delta$ is the global transition function of $\mathcal{C}$. 
Corollary 3 (Topological Variant; Morton Landers Curtis, Gustav Arnold Hedlund, and Roger Conant Lyndon, 1969). Let $\mathcal{R}=\left((M, G, \triangleright),\left(m_{0}\right.\right.$, $\left.\left.\left\{g_{m_{0}, m}\right\}_{m \in M}\right)\right)$ be a cell space, let $Q$ be a finite set, let $\Delta$ be a map from $Q^{M}$ to $Q^{M}$, let $Q^{M}$ be equipped with the prodiscrete topology, and let $H$ be a subgroup of $G$ such that $\left\{g_{m_{0}, m} \mid m \in M\right\} \subseteq H$. The following statements are equivalent:

1. The map $\Delta$ is the global transition function of a semi-cellular automaton with

- $H_{0}$-invariant local transition function and finite essential neighbourhood.

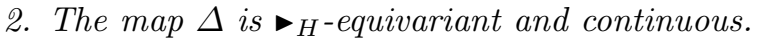

Proof. With Remark 5 this follows directly from Main Theorem 5.

Remark 6 . In the case that $M=G$ and $\triangleright$ is the group multiplication of $G$, Main Theorem [5] is Theorem 1.9.1 in [1] and Corollary 3 is Theorem 1.8.1 in [1].

\section{Invertibility}

Definition 25. Let $\mathcal{C}=(\mathcal{R}, Q, N, \delta)$ be a semi-cellular automaton. It is called invertible if and only if there is a semi-cellular automaton $\mathcal{C}^{\prime}$, called inverse to $\mathcal{C}$, such that the global transition functions of $\mathcal{C}$ and $\mathcal{C}^{\prime}$ are inverse to each other.

Theorem 6. Let $\mathcal{R}=\left((M, G, \triangleright),\left(m_{0},\left\{g_{m_{0}, m}\right\}_{m \in M}\right)\right)$ be a semi-proper cell space, let $Q$ be a set, let $\Delta$ be a map from $Q^{M}$ to $Q^{M}$, let $Q^{M}$ be equipped with the uniformity of discrete convergence on compacta, and let $H$ be a subgroup of $G$ such that $\left\{g_{m_{0}, m} \mid m \in M\right\} \subseteq H$. The following statements are equivalent:

1. The map $\Delta$ is the global transition function of an invertible semi-cellular automaton $\mathcal{C}$ that has an inverse $\mathcal{C}^{\prime}$ such that the local transition functions of $\mathcal{C}$ and $\mathcal{C}^{\prime}$ are $\bullet_{H_{0}}$-invariant, and $\mathcal{C}$ and $\mathcal{C}^{\prime}$ have compact essential neighbourhoods.

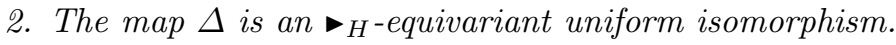

Proof. With Lemma 2 this follows directly from Main Theorem 5

Corollary 4. Let $\mathcal{R}=\left((M, G, \triangleright),\left(m_{0},\left\{g_{m_{0}, m}\right\}_{m \in M}\right)\right)$ be a cell space, let $Q$ be a finite set, let $\Delta$ be a map from $Q^{M}$ to $Q^{M}$, let $Q^{M}$ be equipped with the prodiscrete topology, and let $H$ be a subgroup of $G$ such that $\left\{g_{m_{0}, m} \mid m \in M\right\} \subseteq H$. The following statements are equivalent:

1. The map $\Delta$ is the global transition function of an invertible semi-cellular automaton $\mathcal{C}$ that has an inverse $\mathcal{C}^{\prime}$ such that the local transition functions of $\mathcal{C}$ and $\mathcal{C}^{\prime}$ are $\bullet_{H_{0}}$-invariant, and $\mathcal{C}$ and $\mathcal{C}^{\prime}$ have finite essential neighbourhoods.

2. The map $\Delta$ is $\boldsymbol{H}_{\text {-equivariant, continuous, and bijective. }}$

Proof. With Remark 5 this follows directly from Theorem 6 .

Corollary 5. Let $\mathcal{R}=\left((M, G, \triangleright),\left(m_{0},\left\{g_{m_{0}, m}\right\}_{m \in M}\right)\right)$ be a cell space, let $H$ be a subgroup of $G$ such that $\left\{g_{m_{0}, m} \mid m \in M\right\} \subseteq H$. Furthermore, let $\mathcal{C}=(\mathcal{R}$, $Q, N, \delta)$ be a semi-cellular automaton with finite set of states, finite essential neighbourhood, and $\bullet_{H_{0}}$-invariant local transition function. The automaton $\mathcal{C}$ is invertible if and only if its global transition function is bijective. 
Proof. With Item 1 of Theorem 2 and Corollary 3 this follows directly from Corollary 4

\section{References}

1. Ceccherini-Silberstein, Tullio and Michel Coornaert. Cellular Automata and Groups. In: Springer Monographs in Mathematics. Springer-Verlag, 2010.

2. Hedlund, Gustav Arnold. Endomorphisms and automorphisms of the shift dynamical system. In: Mathematical systems theory 3.4 (1969), pages 320-375.

3. Sébastien Moriceau. Cellular Automata on a $G$-Set. In: Journal of Cellular Automata 6.6 (2011), pages 461-486.

4. Simon Wacker. Cellular Automata on Group Sets and the Uniform Curtis-HedlundLyndon Theorem. arXiv:1603.07271 [math.GR].

5. Simon Wacker. Right Amenable Left Group Sets and the Tarski-Følner Theorem. arXiv:1603.06460 [math.GR].

6. Simon Wacker. The Garden of Eden Theorem for Cellular Automata on Group Sets. arXiv:1603.07272 [math.GR]. 


\section{A Uniformity}

The theory of uniformities as presented here may be found in more detail in Appendix B in the monograph 'Cellular Automata and Groups' [1].

Definition 26. Let $X$ be a set. The diagonal $\{(x, x) \mid x \in X\}$ in $X \times X$ is denoted by $\Delta_{X}$.

Definition 27. Let $X$ be a set, let $R$ be a subset of $X \times X$, and let $y$ be an element of $X$. The $y$-th column $\{x \in X \mid(x, y) \in R\}$ in $R$ is denoted by $R[y]$.

Definition 28. Let $X$ be a set and let $R$ be a subset of $X \times X$. The inverse $\{(y, x) \mid(x, y) \in R\}$ of $R$ is denoted by $R^{-1}$. The set $R$ is called symmetric if and only if it is self-inverse, that is, $R^{-1}=R$.

Definition 29. Let $X$ be a set, and let $R$ and $R^{\prime}$ be two subsets of $X \times X$. The composite $\left\{(x, z) \mid \exists y \in X:(x, y) \in R \wedge(y, z) \in R^{\prime}\right\}$ is denoted by $R \circ R^{\prime}$.

Definition 30. Let $X$ be a set and let $\mathcal{U}$ be a set of subsets of $X \times X$. The set $\mathcal{U}$ is called uniformity on $X$ if and only if

$$
\begin{gathered}
\mathcal{U} \neq \emptyset, \\
\forall E \in \mathcal{U}: \Delta_{X} \subseteq E, \\
\forall E \in \mathcal{U} \forall E^{\prime} \subseteq X \times X:\left(E \subseteq E^{\prime} \Longrightarrow E^{\prime} \in \mathcal{U}\right), \\
\forall E \in \mathcal{U} \forall E^{\prime} \in \mathcal{U}: E \cap E^{\prime} \in \mathcal{U}, \\
\forall E \in \mathcal{U}: E^{-1} \in \mathcal{U}, \\
\forall E \in \mathcal{U} \exists E^{\prime} \in \mathcal{U}: E^{\prime} \circ E^{\prime} \subseteq E .
\end{gathered}
$$

Definition 31. Let $X$ be a set and let $\mathcal{U}$ be a uniformity on $X$. The tuple $(X, \mathcal{U})$ is called uniform space and each element $E \in \mathcal{U}$ is called entourage of $X$.

Example 16. Let $X$ be a set and let $\mathcal{U}$ be the set of all subsets of $X \times X$ that contain $\Delta_{X}$. Then, $\mathcal{U}$ is the largest uniformity on $X$. Itself as well as the uniform space $(X, \mathcal{U})$ are called uniformly discrete.

Definition 32. Let $(X, \mathcal{U})$ be a uniform space. The set

$$
\{O \subseteq X \mid \forall x \in O \exists E \in \mathcal{U}: E[x]=O\}
$$

is a topology on $X$ and called induced by $\mathcal{U}$.

Example 1\%. Let $(X, \mathcal{U})$ be a discrete uniform space. The topology induced by $\mathcal{U}$ is the discrete topology on $X$.

Definition 33. Let $(X, \mathcal{U})$ be a uniform space and let $\mathcal{B}$ be a subset of $\mathcal{U}$.

1. The set $\mathcal{B}$ is called base of $\mathcal{U}$ if and only if

$$
\forall E \in \mathcal{U} \exists B \in \mathcal{B}: B \subseteq E .
$$


2. The set $\mathcal{B}$ is called subbase of $\mathcal{U}$ if and only if the set

$$
\left\{\bigcap_{i=1}^{n} B_{i} \mid B_{i} \in \mathcal{B}, i \in\{1,2, \ldots, n\}, n \in \mathbb{N}\right\}
$$

is a base of $\mathcal{U}$.

Lemma 16. Let $X$ be a set and let $\mathcal{B}$ be a set of subsets of $X \times X$.

1. The set $\mathcal{B}$ is a base of a uniformity on $X$ if and only if

$$
\begin{gathered}
\mathcal{B} \neq \emptyset, \\
\forall B \in \mathcal{B}: \Delta_{X} \subseteq B, \\
\forall B \in \mathcal{B} \forall B^{\prime} \in \mathcal{B} \exists B^{\prime \prime} \in \mathcal{B}: B^{\prime \prime} \subseteq B \cap B^{\prime}, \\
\forall B \in \mathcal{B} \exists B^{\prime} \in \mathcal{B}: B^{\prime} \subseteq B^{-1}, \\
\forall B \in \mathcal{B} \exists B^{\prime} \in \mathcal{B}: B^{\prime} \circ B^{\prime} \subseteq B .
\end{gathered}
$$

2. The set $\mathcal{B}$ is a subbase of a uniformity on $X$ if and only if

$$
\begin{gathered}
\mathcal{B} \neq \emptyset, \\
\forall B \in \mathcal{B}: \Delta_{X} \subseteq B, \\
\forall B \in \mathcal{B} \exists B^{\prime} \in \mathcal{B}: B^{\prime} \subseteq B^{-1}, \\
\forall B \in \mathcal{B} \exists B^{\prime} \in \mathcal{B}: B^{\prime} \circ B^{\prime} \subseteq B .
\end{gathered}
$$

Definition 34. Let $(X, \mathcal{U})$ and $\left(X^{\prime}, \mathcal{U}^{\prime}\right)$ be two uniform spaces and let $f$ be a map from $X$ to $X^{\prime}$. The map $f$ is called uniformly continuous if and only if

$$
\forall E^{\prime} \in \mathcal{U}^{\prime} \exists E \in \mathcal{U}:(f \times f)(E) \subseteq E^{\prime},
$$

where

$$
\begin{aligned}
f \times f: X \times X & \rightarrow X^{\prime} \times X^{\prime}, \\
\left(x_{1}, x_{2}\right) & \mapsto\left(f\left(x_{1}\right), f\left(x_{2}\right)\right) .
\end{aligned}
$$

Lemma 17. Let $(X, \mathcal{U})$ and $\left(X^{\prime}, \mathcal{U}^{\prime}\right)$ be two uniform spaces, let $f$ be a map from $X$ to $X^{\prime}$, and let $\mathcal{B}^{\prime}$ be a base or subbase of $\mathcal{U}^{\prime}$. The map $f$ is uniformly continuous if and only if

$$
\forall E^{\prime} \in \mathcal{B}^{\prime}:(f \times f)^{-1}\left(E^{\prime}\right) \in \mathcal{U} .
$$

Corollary 6. Let $(X, \mathcal{U})$ and $\left(X^{\prime}, \mathcal{U}^{\prime}\right)$ be two uniform spaces, let $f$ be a map from $X$ to $X^{\prime}$, and let $\mathcal{B}$ and $\mathcal{B}^{\prime}$ be two bases or subbases of $\mathcal{U}$ and $\mathcal{U}^{\prime}$ respectively. The map $f$ is uniformly continuous if and only if

$$
\forall E^{\prime} \in \mathcal{B}^{\prime} \exists E \in \mathcal{B}:(f \times f)(E) \subseteq E^{\prime} .
$$


Lemma 18. Let $(X, \mathcal{U})$ and $\left(X^{\prime}, \mathcal{U}^{\prime}\right)$ be two uniform spaces and let $f: X \rightarrow X^{\prime}$ be a uniformly continuous map. The map $f$ is continuous, where $X$ and $X^{\prime}$ are equipped with the topologies induced by $\mathcal{U}$ and $\mathcal{U}^{\prime}$ respectively.

Theorem 7. Let $(X, \mathcal{U})$ and $\left(X^{\prime}, \mathcal{U}^{\prime}\right)$ be two uniform spaces such that $X$, equipped with the topology induced by $\mathcal{U}$, is compact. Each continuous map $f: X \rightarrow X^{\prime}$ is uniformly continuous.

Definition 35. Let $(X, \mathcal{U})$ and $\left(X^{\prime}, \mathcal{U}^{\prime}\right)$ be two uniform spaces and let $f$ be a map from $X$ to $X^{\prime}$. The map $f$ is called uniform isomorphism if and only if it is bijective, and both $f$ and $f^{-1}$ are uniformly continuous.

Lemma 19. Let $(X, \mathcal{U})$ and $\left(X^{\prime}, \mathcal{U}^{\prime}\right)$ be two uniform spaces such that $X$, equipped with the topology induced by $\mathcal{U}$, is compact and $X^{\prime}$, equipped with the topology induced by $\mathcal{U}^{\prime}$, is Hausdorff. Each continuous and bijective map $f: X \rightarrow X^{\prime}$ is a uniform isomorphism. 